\title{
Infrared emission towards SN 1987A 11 years after outburst: Measurements with ISOCAM ${ }^{\star}$
}

\author{
Jg. Fischera, R. J. Tuffs, and H. J. Völk \\ Max-Planck-Institut für Kernphysik, Saupfercheckweg 1, 69115 Heidelberg, Germany \\ Received 17 August 2001 / Accepted 8 February 2002

\begin{abstract}
We present measurements of the mid-infrared (MIR) emission from SN 1987A, made using the Infrared Space Observatory (ISO) 11 years after outburst. They are the only late epoch detections of this source in the thermal IR regime. The position of the source, determined from an offset to an IR-emitting star, suggests that the emission is associated with SN 1987A or its extended supernova remnant (SNR). A predominantly circumstellar origin is however suggested by the size and orientation of the IR-emitting region, which is comparable with the extension of the inner ring seen with the Hubble Space Telescope (HST). The emission is most probably from is consistent with the hypothesis that the blast wave was propagating into material of moderate density interior to the thick inner ring at the epoch of the ISOCAM observations.
\end{abstract} \\ collisionally-heated circumstellar grains embedded in shocked gas downstream of the blast wave. The MIR extent
}

Key words. stars: supernovae: individual: SN 1987A - ISM: dust - infrared: ISM - supernova remnants

\section{Introduction}

Supernovae are thought to play an important role in the life cycle of interstellar dust grains. On the one hand, shocks driven by supernovae into the interstellar medium (ISM) are considered to be responsible for the destruction of interstellar grains (see e.g. Shull 1977; Draine \& Salpeter 1979; Tielens et al. 1994; Jones et al. 1994). On the other hand, supernovae have also been proposed as sources of interstellar dust (e.g. Hoyle \& Wickramasinghe 1970; Dwek \& Scalo 1980).

SN 1987A in the Large Magellanic Cloud (LMC) was the first supernova in which the condensation of dust in the central metal-rich part of the expanding ejecta has been observed. The appearance of a continuum emission in the IR in the spectrum of SN 1987A 614 days after outburst is attributed to grains formed after at least 500 days (e.g. Wooden 1997). The infrared emission from the condensates was (maybe except at the onset of dust formation; Roche et al. 1993) optically thick at the early epochs when the IR-emission was still bright enough to be observable from ground. Therefore, it was not possible to get clear information about their properties and in particular their mass.

Send offprint requests to: Jg. Fischera, e-mail: Joerg.Fischera@mpi-hd.mpg.de

* Based on observations with ISO, an ESA project with instruments funded by ESA member states (especially the $\mathrm{P} / \mathrm{I}$ countries France, Germany, The Netherlands and the UK) with participation of $I S A S$ and $N A S A$.
Observations with the HST have revealed a bipolar ring structure consisting of a thick inner ring centred at the position of the supernova, and two thin outer rings of larger radius at each side (Burrows et al. 1995). Radio and X-ray observations suggest that, after an initial period of free expansion, the blast wave started to propagate through a region of moderate density with $n_{\mathrm{H}} \approx 100 \mathrm{~cm}^{-3}$ interior to the thick inner ring (Chevalier \& Dwarkadas 1995). Chevalier \& Dwarkadas suggested this region to be an HII region, illuminated by the blue supergiant supernova progenitor and composed of wind material ejected during the prior red supergiant phase.

If the HII region contains dust, extended thermal emission from collisionally heated grains embedded in the shocked gas downstream of the blast wave is to be expected. The presence of circumstellar grains was already invoked at early epochs to account for observations of a prompt echo effect in the mid-infrared (Rank et al. 1988; Chalabaev et al. 1989). It has also been suggested that circumstellar grains can account for observations after 580 days showing an extension of 1".5 at $9 \mu \mathrm{m}$ (Roche et al. 1993). Light echos in the optical have further been used to reconstruct a three dimensional model of the larger scale structures around SN 1987A (Crotts et al. 1995).

The ISO mission (Kessler et al. 1996) gave a unique opportunity to probe the early evolution of SN 1987A in the IR prior to the envelopment of the thick inner ring by the blast wave. In this paper we present sensitive 
measurements made with the ISOCAM instrument (Cesarsky et al. 1996) on board ISO 11 years after outburst. Thus far, these measurements constitute the only late epoch detection of the source in the IR regime. Preliminary results were presented by Tuffs et al. (1998). Here we demonstrate that the detected IR emission is most probably of circumstellar origin.

The paper is structured as follows: in Sect. 2 we present the ISOCAM measurements and describe the reduction and calibration of the data. In Sect. 3 we derive the flux density, position, orientation and size of the mid-infrared (MIR) source, showing that the IR emission from the remnant is resolved, with an extent comparable to that of the thick inner ring. These results are discussed in Sect. 4. A summary of the paper is given in Sect. 5 .

Throughout the paper we adopt a distance of $51 \mathrm{kpc}$ to the supernova as was used to analyse the X-ray observations by Hasinger et al. (1996). This is close to the distance of $51.2 \pm 3.1 \mathrm{kpc}$ derived by Panagia et al. (1991) but slightly larger than the values given in later publications (Gould 1995; Sonneborn et al. 1997; Gould \& Uza 1998).

\section{Observations}

The observations were made with the ISOCAM instrument in the $L W 2, L W 10$ and $L W 3$ broad-band filters, whose pass bands are centred on $6.75,12$ and $14.3 \mu \mathrm{m}$ respectively. An observation log summarizing the principal observational parameters is given in Table 1.

The measurements in $L W 10$ and $L W 3$ were optimized for obtaining sensitive photometry and structural information of a faint compact target. A pixel field of view (PFOV) of $3^{\prime \prime}$ was chosen as for the anticipated backgrounds it gave the best trade off between angular resolution and detector illumination. We used a $3 \times 3$ raster with raster interval $10^{\prime \prime} \times 10^{\prime \prime}$ to sample the sky at intervals of $1^{\prime \prime}$. This comfortably oversampled the point spread function (psf), which has a $F W H M$ of $\sim 4^{\prime \prime}$ at $10 \mu \mathrm{m}$ for the $3^{\prime \prime}$ PFOV.

The raster interval of $10^{\prime \prime}$ was the minimum interval that provides a sky sampling for which each view of the target in a given pixel is immediately preceded or followed by a view of the underlying background. This optimized the knowledge of the relative response of the detector between source and background, which in turn could be used to minimize the effect of residual uncertainties in the flatfield response of the $32 \times 32$ pixel detector on the determination of source structure. The observations were separated in epoch over the period September 1997 to February 1998. The measurement in the $12 \mu \mathrm{m}$ band was a follow up observation to the detection in the $14.3 \mu \mathrm{m}$, while the measurement in the $6.75 \mu \mathrm{m}$ band was part of an independent program of large-scale mapping of the LMC led by L. Vigroux.
Table 1. Observation log.

\begin{tabular}{l||c|c|c}
\hline \hline Filter & $L W 2$ & $L W 10$ & $L W 3$ \\
\hline TDT & 667017 & 811022 & 750019 \\
observer & L. Vigroux & R.J. Tuffs & R.J. Tuffs \\
$\lambda_{\text {ref }}[\mu \mathrm{m}]$ & 6.75 & 12. & 14.3 \\
filter range $[\mu \mathrm{m}]$ & $5.00-8.50$ & $8.00-15.00$ & $12.0-18$. \\
observation date & 13.9 .97 & 5.12 .97 & 3.2 .98 \\
day after outburst & 3855.2 & 3998.7 & 3937.7 \\
raster & $2 \times 2$ & $3 \times 3$ & $3 \times 3$ \\
pixel size & $6^{\prime \prime}$ & $3^{\prime \prime}$ & $3^{\prime \prime}$ \\
step size & $24^{\prime \prime}$ & $10^{\prime \prime}$ & $10^{\prime \prime}$ \\
read out interval $t_{\text {int }}$ & $2.10 \mathrm{~s}$ & $5.04 \mathrm{~s}$ & $5.04 \mathrm{~s}$ \\
reads per pointing $n_{\mathrm{i}}$ & 31 & 19 & 19 \\
\hline
\end{tabular}

${ }^{a}$ The time of the outburst is taken to be February 23.316, 1987 (Arnett et al. 1989).

\subsection{Calibration and data reduction}

The data were first corrected for dark current using the model of the Cam Interactive Analysis (CIA) package (Ott et al. 1998; version April 2000). This was followed by glitch removal using the Multi resolution Median Transform, also provided by CIA. The data were then inspected and residual events and strong longer-lived glitchinduced distortions were removed manually. To allow an accurate determination of the illumination seen by each single pixel during the raster, the data were then corrected for the transient response behavior of the detector. To this end we developed and implemented a routine based on the drift model of the ISOPHOT-S detector on board ISO (Schubert 1995). The routine derived the time constants and jump factors determining the drift behaviour of the individual detector pixels from the measured data.

To create a map showing the background structure underlying the sources we applied a flat-field correction, which was optimized for the smooth variation of the background. The flat-field was derived from a map, which had already been corrected using a library flat provided by CIA and which had been spatially filtered to reduce the noise. The flat-field-corrected data were then binned (without interpolation) on a grid with a sampling of $1^{\prime \prime}$. For visualization the data were further filtered by setting pixel values differing more than one sigma from their surroundings to the average value of the surrounding pixels (the filter was not applied for obvious discrete sources). Then map pixels without any data were set to the average values of the neighbouring pixels. Finally, we convolved the image with a Gauss function with a variance of 0 .' 6 . The resulting maps are shown in Fig. 1.

The quantitative analysis of the individual compact sources seen in the ISOCAM maps was done directly from the observed brightnesses as a function of sky position, without use of maps, as described in Sect. 3. This required a different calibration procedure compared to that for the maps. For the observations at 12 and $14.3 \mu \mathrm{m}$ we made use of the almost flat emission around the two sources and normalized all pixel values at individual raster positions with the corresponding values of the background. 

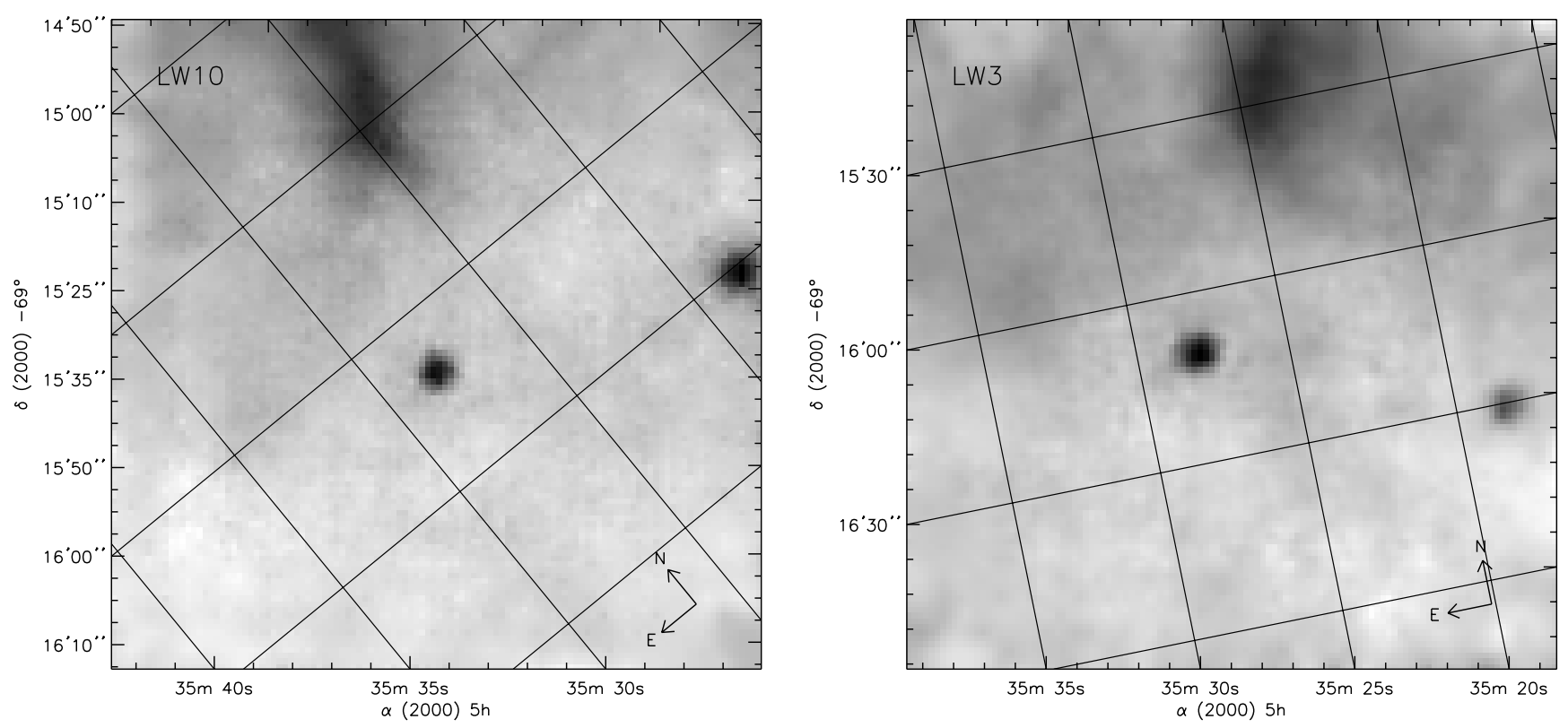

Fig. 1. Region around the SN 1987A in the MIR, as seen at $12 \mu \mathrm{m}(L W 10)$ and $14.3 \mu \mathrm{m}(L W 3)$. The brightness increases with darkness and ranges from 0.215 to $0.306 \mathrm{mJy} /\left[^{\prime \prime}\right]^{2}(L W 10)$ or from 0.356 to $0.426 \mathrm{mJy} /\left[^{\prime \prime}\right]^{2}$ (LW3) respectively. The source close to the middle is emission from the remnant of SN 1987A. The IR-emission at the border of the image is the reference star, that we used to derive the absolute coordinates of the IR-emission from the remnant of SN 1987A. The bright emission in the north is an arm of 30 Doradus. The two individual sources show different colours, suggesting a lower temperature for the IR-emission from SN 1987A than for the star.

This was done by making a linear fit to the variation of (responsivity drift-corrected) illumination in each given detector pixel with spacecraft raster position, leaving out raster positions where the pixel was viewing the source. In the case of the measurement at $6.75 \mu \mathrm{m}$, we used the library flat provided in CIA, as the raster pattern was not suitable for this method. For the measurement at 12 and at $14.3 \mu \mathrm{m}$ the sky coordinates of each detector pixel at each raster pointing were evaluated from the actual (post posteri) pointing position of the satellite using the median of all the data used. To derive the pixel coordinates of the observation at $6.75 \mu \mathrm{m}$ we made use of the astronomical information provided by the CIA-program. Corrections were applied for the astrometrical distortions in the ISOCAM field (Aussel 1998) induced by the field lens using the polynomial correction coefficients for the different filters as given in CIA.

\subsection{The ISOCAM maps}

The final images show two clear individual sources. The source close to the middle corresponds to emission from the remnant of SN 1987A, whereas the source near the edge is an IR-emitting star. The two sources show different colours, indicating a higher temperature for the star. The serendipitous detection of the star provided a unique possibility to determine an accurate position for the central MIR source near SN 1987A (see Sect. 3.3). The bright ridge of emission to the NNW of SN 1987A is an arm of the 30 Doradus nebula. Although backgrounds are dominated by smooth emission from zodiacal light, there is some evidence for faint cirrus structure at the $1 \%$ level in the southern part of the field. At both wavelengths the IR emission near the map centre is offset several arcsec from the nominal position of SN 1987A. These offsets are partly due to the inaccuracy of the position of the satellite but are mainly attributable to a displacement of the field lens of the camera (Blommaert \& Cesarsky 1998).

\section{Parameters of the discrete sources}

Various parameters of the discrete sources, expressed as the vector $\boldsymbol{a}$, were derived using a non-linear $\chi^{2}$-fit, where we compared the measured data $f\left(\boldsymbol{x}_{i}\right)$ and uncertainties $\sigma_{i}$ at the sky positions $\boldsymbol{x}_{i}$ around the individual sources with a model image $B\left(\boldsymbol{x}_{i}, \boldsymbol{a}\right)$ :

$\chi^{2}(\boldsymbol{a})=\sum_{i} \frac{\left(B\left(\boldsymbol{x}_{i}, \boldsymbol{a}\right)-f\left(\boldsymbol{x}_{i}\right)\right)^{2}}{\sigma_{i}^{2}}$.

The model image $B(\boldsymbol{x}, \boldsymbol{a})$ is in general the convolution of the theoretical psf corresponding to the telescope optics and the spectral response of the used filter band pass with the source function (taken to be a Gaussian), on a certain background. For a symmetrical Gaussian fit $\boldsymbol{a}$ is $\left[F_{\mathrm{Q}}, \boldsymbol{x}_{0}, \sigma_{\mathrm{Q}}, I_{\mathrm{U}}\right]$ and the model image given by:

$$
\begin{aligned}
B(\boldsymbol{x}, \boldsymbol{a})= & I_{\mathrm{U}}+\int J_{\mathrm{F}}\left(\boldsymbol{x}-\boldsymbol{x}^{\prime}\right) \\
& \times \frac{F_{\mathrm{Q}}}{2 \pi \sigma_{\mathrm{Q}}^{2}} \exp \left(-\frac{\left(\boldsymbol{x}^{\prime}-\boldsymbol{x}_{0}\right)^{2}}{2 \sigma_{\mathrm{Q}}^{2}}\right) \mathrm{d} \boldsymbol{x}^{\prime},
\end{aligned}
$$

where $J_{\mathrm{F}}(\boldsymbol{x})$ is the theoretical broad-band psf (see Appendix A), $F_{\mathrm{Q}}$ the flux density, $\boldsymbol{x}_{0}$ the coordinates, 


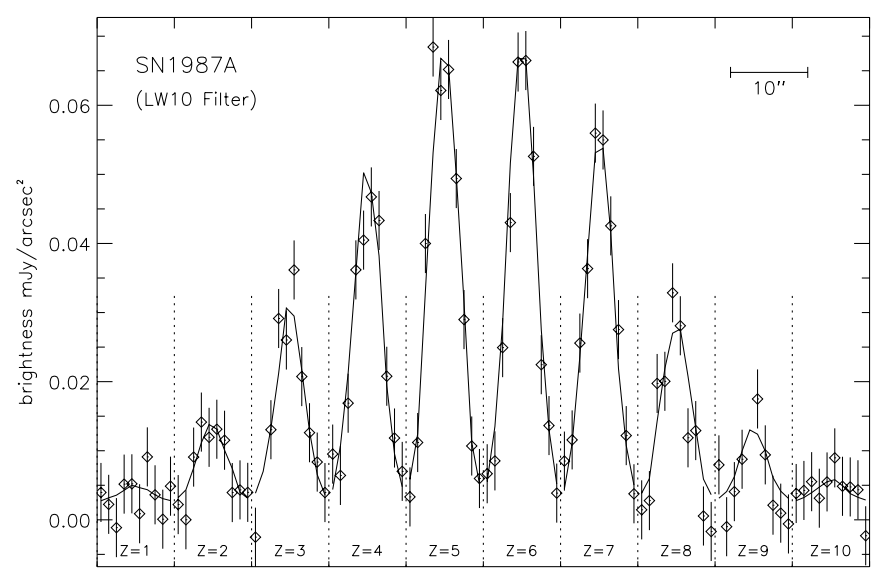

position in $Y$

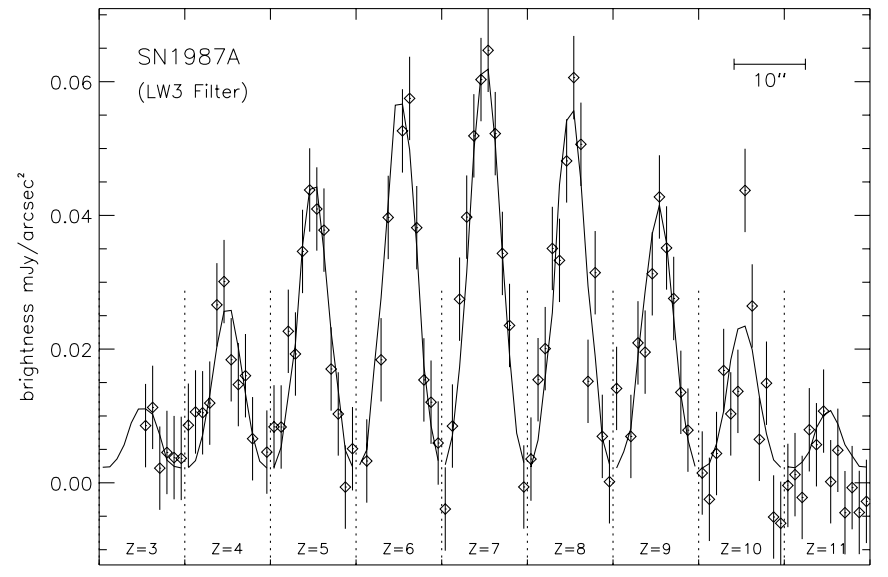

position in $Y$

Fig. 2. Best fits of the theoretical image (solid line) to the measured brightnesses (diamonds) of the remnant of SN 1987A at $12 \mu \mathrm{m}(L W 10)$ and $14.3 \mu \mathrm{m}(L W 3)$. They are shown as scans along lines (at fixed $Z$ in the coordinate system of the satellite) through a subimage centred on SN 1987A. The dotted vertical lines mark the beginning and the end of the scans. To calculate the $p f$ for each filter the spectral shape was assumed to be $F_{\lambda} \propto \lambda^{-k}$ with a spectral index $k=1.08$. The $F W H M$ of the circular Gaussian source is 1 .' $50(L W 10)$ and 1 .' 13 ( $L W 3)$.

$\sigma_{\mathrm{Q}}$ the variance of the Gaussian source and $I_{\mathrm{U}}$ the background. The expression 1 was minimized to derive $F_{\mathrm{Q}}, \boldsymbol{x}_{0}$, $I_{\mathrm{U}}$ (at $6.75 \mu \mathrm{m}$ only) and $\sigma_{\mathrm{Q}}$ (only at 12 and $14.3 \mu \mathrm{m}$ ).

To achieve a higher accuracy, in particular for the determination of the size of the emitting region, we took into account the dependence of beam profile on source colour. This was done by averaging simulated beam shapes obtained at a series of wavelengths across the broad filter pass bands, weighted according to a power law source spectrum $F_{\lambda} \lambda^{k}=$ const. with a spectral index $k$ (see Appendix A). For the star the spectral index was always taken to be $k=4$ appropriate to a Rayleigh-Jeans spectrum. For the source associated with the supernova $k$ was estimated from the finally determined colour corrected (see e.g. Blommaert 1998) flux densities to be $1.08 \pm 0.32$. The model image was calculated on a grid with a resolution of $1 / 3^{\prime \prime}$. In the fitting procedure the predicted fluxes at the observed positions were found using linear interpolation.

The true uncertainties $\sigma_{i}$ in each individual measurement for a detector pixel at a given spacecraft raster pointing direction are difficult to estimate and may be partly affected by systematic uncertainties. This particularly applies to measurements viewing the discrete sources, where the illumination and detector response function may vary with position over the field of view of the individual detector pixels. We therefore assumed in nearly all model calculations that the uncertainties of all measurements are equal. This is justified by the fact that the distribution of the residuals was found to be normal. The only exception to this was made for the measurement of the star at $6.75 \mu \mathrm{m}$, where the uncertainties of the high source fluxes were mainly attributed to a particularly strong transient behaviour.

The absolute uncertainties $\sigma_{i}$ of the measured data were chosen such that the value of the minimum $\chi^{2}$
(Eq. (1)) corresponds to a confidence level of $90 \%$. In the case of the measurements at 12 and at $14.3 \mu \mathrm{m}$ we made a special fit letting $F_{\mathrm{Q}}, \boldsymbol{x}_{0}, \sigma_{\mathrm{Q}}$ and $k$ as free variables. The joint uncertainties of $\nu$ parameters were estimated by finding the range within which the function $\Delta \chi_{\nu}^{2}=\chi_{\nu}^{2}-\chi_{\min }^{2}$ did not exceed the value corresponding to a confidence level of one sigma, letting all other parameters as free variables. Thus, apart from the jointly determined uncertainties in the coordinates, which were estimated from $\Delta \chi_{2}^{2}=2.30$, the uncertainties in individual parameters correspond to $\Delta \chi_{1}^{2}=1$ (see e.g. Press et al. 1992).

\subsection{Fits with a symmetrical Gaussian as IR-source}

In the case of the measurement at $6.75 \mu \mathrm{m}$ the parameters varied were $F_{\mathrm{Q}}$, the coordinates $\boldsymbol{x}_{0}$ and the background brightness $I_{\mathrm{U}}$. We used a model image corresponding to an ideal point source $\left(\sigma_{\mathrm{Q}}=0\right)$ assuming a spectrum with $k=1$ for the IR emission from the supernova remnant.

At 12 and at $14.3 \mu \mathrm{m}$ the flux density and the coordinates were optimized together with the extension of the source. The variables varied were therefore $F_{\mathrm{Q}}, \boldsymbol{x}_{0}$ and $\sigma_{\mathrm{Q}}$. Because of the normalization of the pixel data to the background we held $I_{\mathrm{U}}$ fixed. The uncertainties in $F_{\mathrm{Q}}$ and $\boldsymbol{x}_{0}$ were estimated for a fixed (most probable) source size. The best fits to the measured data with $L W 10$ and $L W 3$ are shown in Fig. 2.

The surface brightness of the background at these two wavelengths was estimated from the images calculated as described in Sect. 2.1, but without filtering and convolution. To derive the surface brightness $I_{U}$ at the position of the supernova from the maps we took the average value within two circular areas of radius of $5^{\prime \prime}$ offset $15^{\prime \prime}$ to the east and to the west of SN 1987A. At both 12 and $14.3 \mu \mathrm{m}$ the difference between the two values is less than $1 \%$. For 
Table 2. Spherical Gauss fits to the IR emission associated with SN 1987A and the reference star.

\begin{tabular}{|c|c|c|c|c|c|c|}
\hline & \multicolumn{3}{|c|}{$\overline{\mathrm{SN} 1987 \mathrm{~A}}$} & \multicolumn{3}{|c|}{ reference star } \\
\hline$\overline{\text { Filter }}$ & $L W 2$ & $L W 10$ & $L W 3$ & $\| L W 2$ & $L W 10$ & $L W 3$ \\
\hline$\lambda_{\text {ref }}[\mu \mathrm{m}]$ & 6.75 & 12. & 14.3 & |6.75 & 12. & 14.3 \\
\hline $\mathrm{ADU}^{a} /(\mathrm{mJy} / \mathrm{pixel})^{b}$ & $2.32 \pm 3.3 \%$ & $4.23 \pm 3.9 \%$ & $1.96 \pm 4.8 \%$ & $2.32 \pm 3.3 \%$ & $4.23 \pm 3.9 \%$ & $1.96 \pm$ \\
\hline beam size $\left(F W H M\left[^{\prime \prime}\right]\right)^{c}$ & $6.887 \pm 0.002$ & $4.195 \pm 0.025$ & $5.109 \pm 0.009$ & $6.222 \pm 0.001$ & $3.945 \pm 0.026$ & $4.955 \pm 0.008$ \\
\hline spectral index $k^{d}$ & 1. & 1.08 & 1.08 & || 4 & 4 & 4 \\
\hline derived source size $\left(F W H M\left[^{\prime \prime}\right]\right)^{e}$ & - & $1.50_{-0.24}^{+0.21}$ & $<2.3(3 \sigma)$ & I- & $2.26_{-0.30}^{+0.27}$ & $<2.4(3 \sigma)$ \\
\hline$\chi_{\nu}^{2}(\nu)$ & $0.76(51)$ & $0.82(94)$ & $0.83(127)$ & $0.77(60)$ & $0.69(41)$ & $0.78(75)$ \\
\hline Uncertainty of pixel values $\sigma / I_{\mathrm{U}}$ & $3.6 \%$ & $1.9 \%$ & $1.7 \%$ & $12 . \%^{f}$ & $2.5 \%$ & $1.5 \%$ \\
\hline$F_{\mathrm{Q}} / I_{\mathrm{U}}\left[\left[^{\prime \prime}\right]^{2}\right]$ & $6.54 \pm 25 \%$ & $10.62 \pm 1.8 \%$ & $7.608 \pm 2.6 \%$ & $116.2 \pm 5.5 \%$ & $16.11 \pm 3.1 \%$ & $4.879 \pm 5.0 \%$ \\
\hline background $I_{\mathrm{U}}\left[\mathrm{mJy} /\left[^{\prime \prime}\right]^{2}\right]$ & $0.0759 \pm 3.3 \%$ & $0.226 \pm 4.0 \%$ & $0.370 \pm 4.8 \%$ & $0.0717 \pm 3.6 \%$ & $0.228 \pm 6.1 \%$ & $0.365 \pm 5.0 \%$ \\
\hline source flux density $F_{\mathrm{Q}}[\mathrm{mJy}]^{g}$ & $0.50 \pm 25 \%$ & $2.40 \pm 4.3 \%$ & $2.82 \pm 5.5 \%$ & $\mid 8.33 \pm 6.2 \%$ & $3.67 \pm 5.0 \%$ & $1.78 \pm 7.0 \%$ \\
\hline source flux density $F_{Q}^{\text {corr }}[\mathrm{mJy}]^{h}$ & $0.43 \pm 25 \%$ & $2.64 \pm 4.3 \%$ & $2.90 \pm 5.5 \%$ & $8.43 \pm 6.2 \%$ & $3.73 \pm 5.0 \%$ & $1.69 \pm 7.0 \%$ \\
\hline
\end{tabular}

${ }^{a}$ Analog to Digital Units.

${ }^{b}$ Blommaert et al. (1999).

${ }^{c}$ Average $F W H M$ of cuts in $Z$ and $Y$ through the maximum of the theoretical psf.

Uncertainties correspond to the difference of these two values.

${ }^{d}$ Spectral index assumed in calculating the theoretical psf (see text).

${ }^{e} F W H M$ of the symmetrical Gaussian model assumed for the source. Uncertainties are given as $1 \sigma$.

${ }^{f}$ Median of the uncertainties.

${ }^{g}$ Flux density without colour correction.

${ }^{h}$ Colour-corrected according to a spectrum $F_{\lambda} \propto \lambda^{-2} B_{\lambda}(T)$ with $T=198.7 \mathrm{~K}$ (supernova) and $T=564.1 \mathrm{~K}$ (star).

the background brightnesses at the position of the star we took the average of a number of single measurements around the star; here the estimated uncertainties in the background are $1.3 \%(L W 10)$ and $0.9 \%(L W 3)$.

The derived values for the source flux and size, and the background brightnesses, are summarized in Table 2, together with the assumed value for the spectral index $k$ used in the determination of source size. The table also gives the source flux densities normalized to the surface brightness of the background. The uncertainties quoted for the normalized flux densities at 12 and $14.3 \mu \mathrm{m}$ are statistical uncertainties derived from the $\chi^{2}$-fit.

The IR spectrum of the supernova remnant can be approximated by a simple black body spectrum with $T=288 \pm 16 \mathrm{~K}\left(\chi_{\min }^{2}=0.84\right)$ or a modified black body spectrum $F_{\lambda} \propto \lambda^{-2} B_{\lambda}(T)$ with $T=198.7_{-7.8}^{+9.9} \mathrm{~K}$ where a good fit is achieved $\left(\chi_{\min }^{2}=0.02\right)$. Assuming the same modified spectrum for the star we get a temperature of $T=564.1_{-25.1}^{+27.2} \mathrm{~K}$. The fit is again very good $\left(\chi_{\min }^{2}=\right.$ $\left.1.2 \times 10^{-3}\right)$. A simple black body for the star seems to be unlikely $\left(\chi_{\min }^{2} \approx 5.3\right)$.

\subsubsection{Sizes of the IR-sources}

The probability distributions for the extension of the IRemitting regions associated with SN 1987A were derived at 12 and at $14.3 \mu \mathrm{m}$ from a $\chi^{2}$-fit using a symmetrical Gaussian as source function (see Appendix B). These are shown in Fig. 3. The different probability distributions correspond to different colour indices used in the calculation of the theoretical $p s f$. The distributions plotted with thick solid lines have been derived for the most probable colour of the source, a power law with $k=1.08$. As can be seen in the figure, the probability distributions are only slightly affected by the uncertainty in the spectral index $k$ for the MIR emission associated with the supernova. The probability distributions corresponding to the upper and lower limits for $k$ (0.56 and 1.59 , respectively, at $90 \%$ confidence level) are plotted as thin solid lines. The dashed and dotted curves peaking near $1^{\prime \prime} 9$ are probability distributions for the measurements at $12 \mu \mathrm{m}$ using as psf the image of a calibration star and the theoretical psf for a spectral index $k=4$, respectively. Since the spectrum of the star should be a power law with $k=4$, the similarity of these two curves demonstrates that the effect of interchanging the theoretical psf with the measured psf is small. The larger apparent source sizes calculated from the psfs with $k=4$ arise from the decrease in $F W H M$ of the $p s f$ with increasing $k$ compared with the true value $k=1.08 \pm 0.32$ for the MIR emission associated with SN 1987A.

For the $L W 10$ observation we obtained a $F W H M$ of $1.50_{-0.24}^{+0.21}$ arcsec for the extension of the source. This size is in agreement with the extensions of the projected inner ring, seen in the HST picture. The distribution we derived from the $L W 3$ observation is broader, and also has a higher probability for small extensions. This might be due to the strong glitches causing long time distortions of the detector response of the observation (see also Sect. 3.2). From this observation we were only able to obtain an upper limit for the source size of 2 .' 3 at $3 \sigma$ level. Table 3 gives the maximum and minimum sizes of the IRemitting region for certain confidence levels derived from the probability distributions for $k=1.08$. As shown in Appendix B.2 the theoretical psf for the $L W 10$ filter is a sufficiently accurate representation of the real psf to 


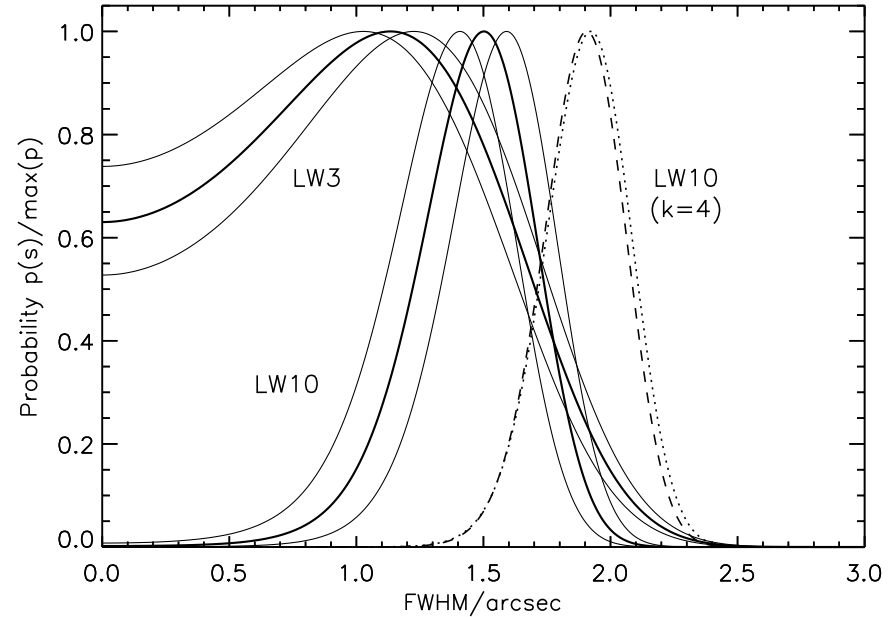

Fig. 3. Derived probability distributions of the size $s$ of the theoretical image (see Eq. (B.1)) as a function of the $F W H M$ of the IR source for the two filter measurements LW10 $(12 \mu \mathrm{m})$ and LW3 $(14.3 \mu \mathrm{m})$ of SN 1987A. The emitting region is assumed to be a circular Gaussian. Also shown is the effect of different source spectra of form $F_{\lambda} \propto \lambda^{-k}$ with spectral index $k$. The probability distributions shown as thick lines correspond to the most probable spectral index $k=1.08$ as derived from the flux densities at 12 and $14.3 \mu \mathrm{m}$. The distributions corresponding to the upper and lower limits for $k$ (0.56 and 1.59 , respectively, at $90 \%$ confidence level) are plotted as thin solid lines. The probability distributions shown as dashed and dotted lines are size measurements of the IR emission associated with the supernova at $12 \mu \mathrm{m}$ using as $p s f \mathrm{~s}$ an image of a calibration star (HIC 78527) and a theoretical psf with $k=4$, respectively.

Table 3. Sizes of the IR source associated with SN 1987A.

\begin{tabular}{l|lllll}
\hline \hline conf. level & $68 \%$ & $90 \%$ & $95 \%$ & $99 \%$ & $99.9 \%$ \\
\hline \hline & \multicolumn{5}{|l}{ Maximum size of the source $(F W H M)$} \\
\hline$L W 3$ & $1^{\prime \prime} .46$ & $1^{\prime \prime} .79$ & $1^{\prime \prime} 92$ & $2^{\prime \prime} 15$ & $2^{\prime \prime} 40$ \\
$L W 10$ & $1^{\prime \prime} 60$ & $1^{\prime \prime} .76$ & $1^{\prime \prime} 83$ & $1^{\prime \prime} 95$ & $2^{\prime \prime} 08$ \\
\hline & \multicolumn{5}{|l}{ Minimum size of the source $(F W H M)$} \\
\hline$L W 3$ & $1^{\prime \prime} .03$ & $0^{\prime \prime} 62$ & $0^{\prime \prime} 45$ & $0^{\prime \prime} 21$ & $0^{\prime \prime} 07$ \\
$L W 10$ & $1^{\prime \prime} 40$ & $1^{\prime \prime} 19$ & $1^{\prime \prime} 09$ & $0^{\prime \prime} 87$ & $0^{\prime \prime} 56$ \\
\hline
\end{tabular}

resolve sources with a $F W H M$ greater than $\sim 0$ "' 6 . If we take the measurement at $12 \mu \mathrm{m}$ the source is extended with a certainty of more than $99 \%$.

Some evidence was found for extended emission associated with the star at $12 \mu \mathrm{m}$ (Table 2 ). This may in part be due to the strong edge position and the course sampling of the star. But it cannot be excluded from the IR observations that the star itself is extended.

\subsection{Fits with an elliptical Gauss function as IR-source}

In addition to the extension we also examined the orientation angle of the IR-source. Herefore we described the source through a two dimensional elliptical Gauss function with variances $\sigma_{\tilde{x}}$ and $\sigma_{\tilde{y}}$ and a rotation angle $\vartheta$, which was taken to be the angle between the negative $Z$-axis in the satellite coordinate system and the $\tilde{y}$-axis of the rotated Gaussian, measured counter clockwise. $\boldsymbol{a}$ is then $\left[F_{\mathrm{Q}}, \boldsymbol{x}_{0}, \sigma_{\tilde{x}}, \sigma_{\tilde{y}}, \vartheta, I_{\mathrm{U}}\right]$ and the model image given by:

$$
\begin{aligned}
& B(\boldsymbol{x}, \boldsymbol{a})=I_{\mathrm{U}}+\int \mathrm{d} \boldsymbol{x}^{\prime}\left\{J_{\mathrm{F}}\left(\boldsymbol{x}-\boldsymbol{x}^{\prime}\right) \frac{F_{\mathrm{Q}}}{2 \pi \sigma_{\tilde{x}} \sigma_{\tilde{y}}}\right. \\
& \left.\quad \times \exp \left(-\frac{\tilde{x}\left(\vartheta, \boldsymbol{x}^{\prime}, \boldsymbol{x}_{0}\right)^{2}}{2 \sigma_{\tilde{x}}^{2}}-\frac{\tilde{y}\left(\vartheta, \boldsymbol{x}^{\prime}, \boldsymbol{x}_{0}\right)^{2}}{2 \sigma_{\tilde{y}}^{2}}\right)\right\},
\end{aligned}
$$

where $\tilde{\boldsymbol{x}}\left(\vartheta, \boldsymbol{x}, \boldsymbol{x}_{0}\right)=\left(\tilde{x}\left(\vartheta, \boldsymbol{x}, \boldsymbol{x}_{0}\right), \tilde{y}\left(\vartheta, \boldsymbol{x}, \boldsymbol{x}_{0}\right)\right)$ are the coordinates of the rotated Gaussian:

$\tilde{\boldsymbol{x}}\left(\vartheta, \boldsymbol{x}, \boldsymbol{x}_{0}\right)=\left(\begin{array}{rr}\cos (\vartheta) & \sin (\vartheta) \\ -\sin (\vartheta) & \cos (\vartheta)\end{array}\right)\left(\boldsymbol{x}-\boldsymbol{x}_{0}\right)$.

As before we used theoretical $p s f \mathrm{~s}$ for the two filters $L W 10$ and $L W 3$ corresponding to $k=1.08$. For each rotation angle $\vartheta$ we derived best fit variances through the minimization of $\chi^{2}(\boldsymbol{a})$ (Eq. (1)), leaving $F_{\mathrm{Q}}$ and $\boldsymbol{x}_{0}$ as free parameters and $I_{\mathrm{U}}$ fixed. The position angle of the elliptical Gaussian is taken to be the angle of the $\tilde{y}$-axis to the northern direction, measured again counter clockwise. The variation of the minimum $\Delta \chi^{2}$ with the position angle for the two filters is shown in Fig. 4, together with the corresponding extensions given as FWHM. The extensions and the position angle are compared with the orientation and the sizes of the minor and the major axis of the projected inner ring of SN 1987A, seen with the WFPC2 of the HST (Burrows et al. 1995). The source sizes of the best fit are shown in Fig. 5b overlaid on an WFPC2-image, where we have centred the ellipses representing the fitted Gaussian source model at the position of the SN 1987A.

The variation in $\Delta \chi^{2}$ is low in the case of the measurement with $L W 10$. But the coincidence of the derived parameters of the extensions and the rotation angle of the two dimensional Gauss function with the parameters of the inner ring are remarkable. The derived region of the IR source at $14.3 \mu \mathrm{m}$ on the other hand seems to have an extension with the dimension of the inner ring only in one direction. In the other direction, the shown extension is the minimum we allowed to avoid inaccuracies in the modeling. We think that this strong asymmetry is artificial and an effect due to strong glitches at the beginning of the $L W 3$ observation.

\subsection{Position of the individual sources}

As an independent property of the IR emission from the remnant of SN 1987A we derived the absolute coordinates relative to the position of the IR-emitting star visible in the two mosaics at 12 and at $14.3 \mu \mathrm{m}$ (Fig. 1). The position of the IR-emitting star itself was not known so that we first had to determine the absolute coordinates of this star (see Appendix C).

We derived the following offset positions of the IR emission from the position of the supernova, where we have taken the coordinates given by Reynolds et al. (1995):

$\Delta \alpha=-0$ ". $04 \pm 0$. '. $24_{\text {stat. }} \pm 0$ ". $42_{\text {sys. }}$,

$\Delta \delta=+0 . .48 \pm 0.0^{\prime \prime} 24_{\text {stat. }} \pm 00^{\prime \prime} 42_{\text {sys. }}$.

(LW3) 

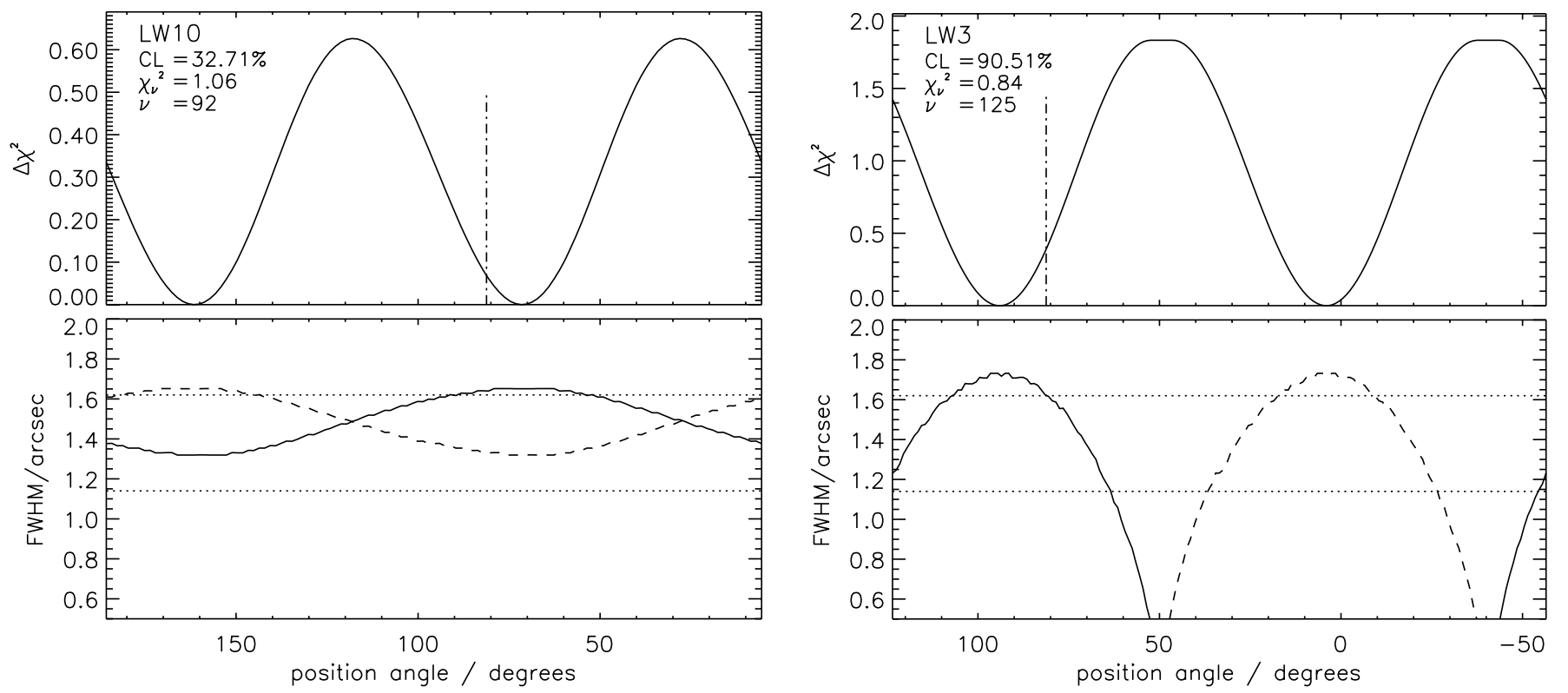

Fig. 4. The top panel shows the variation of $\Delta \chi^{2}$ with the position angle of a two dimensional Gaussian as source function of the IR emission at $12 \mu \mathrm{m}$ (LW10) and $14.3 \mu \mathrm{m}$ (LW3). Below the FWHM of the two directions of the two dimensional Gaussian (solid and dashed line) are shown as function of the position angle. The extensions are compared with the lengths of the two axes of the elliptical projection (dotted lines) of the thick inner ring of SN 1987A, seen with the WFPC2 of the HST (Burrows et al. 1995). The position angle of the major axis of the ring projection $\left(81.2^{\circ}\right)$ is shown in the top panels as the vertical dashed-dotted lines. The given confidence levels belong to the minimum $\Delta \chi^{2}$.

and

$\Delta \alpha=+0^{\prime \prime} 77 \pm 0 . .15_{\text {stat. }} \pm 0^{\prime \prime} \cdot 42_{\text {sys. }}$,

$\Delta \delta=+0^{\prime \prime} 09 \pm 0.0^{\prime \prime} 15_{\text {stat. }} \pm 0 . .42_{\text {sys. }}$.

(LW10)

The systematic uncertainty is not very well known. It is simply assumed that the systematic uncertainty in individual directions is given by the accuracy of the pixel positions in the inner ISOCAM-field after distortion correction, which should be accurate to within a tenth of a pixel (Aussel 1998). The combined uncertainty of the coordinate pair is therefore taken to be $\sim 0^{\prime \prime} 42$. Due to the strong edge position of the star in particular in the $L W 10$-field and the fact, that at one raster point the dead line of the $L W$ camera of the $L W 10$ observation was lying at the sky position of the star, the systematic uncertainty of the offset position at $12 \mu \mathrm{m}$ may be larger.

The absolute coordinates of the IR-emission from the remnant of SN $1987 \mathrm{~A}$ at 12 and $14.3 \mu \mathrm{m}$ with the corresponding uncertainties are shown in Fig. $5 \mathrm{a}$ in relation to the WFPC2-image. Figure 5b shows a visualization of the fits to the size of the emitting region. As seen in Fig. 5a the IR emission measured with ISOCAM should be from the remnant of SN 1987A. Taking the joint probability distribution of both measurements, the most probable location for the IR counterpart is to the north east of the supernova, which is also the position of the maxima in the $\mathrm{X}$-ray and radio surface brightness distributions (Burrows et al. 2000). But it is also possible that the centre of the IR emission lies at the position of the supernova so that the outer boundary of the emitting region could indeed coincide with the inner ring as seen in Fig. 5b. Therefore there is no firm evidence of asymmetry in the MIR emission comparable with that seen in radio and X-ray wavelengths.

\section{Discussion}

Very probably the detected MIR flux densities are dominated by emission from dust grains.

The contribution of synchrotron radiation can be estimated by extrapolating the radio spectrum given by Gaensler et al. (1997) to $10 \mu \mathrm{m}$. Taking into account the linear increase of radio emission with time, this yields the value $\sim 5.1 \times 10^{-3} \mathrm{mJy}$, two orders of magnitude less than the flux densities measured by ISOCAM.

We can also exclude free-free emission from the debris or the thick inner ring as a substantial contributor to the observed MIR flux densities. We have estimated the MIR free-free continuum from optical recombination line measurements using published emissivities and recombination coefficients for case B of Martin (1988).

For the free-free emission from the ejected material we adopted the $\mathrm{H}_{\beta}$ flux 2873 days after outburst (Chugai et al. 1997) assuming a temperature of $10^{3} \mathrm{~K}$, which is consistent with the measured balmer decrement of 3.8. The predicted flux density at $10 \mu \mathrm{m}$ is roughly three orders of magnitudes lower $\left(\sim 3.2 \times 10^{-3} \mathrm{mJy}\right)$. Even in the case of a higher temperature of $10^{4} \mathrm{~K}$ the free-free emission from the supernova debris in the IR is negligible $(\sim 0.027 \mathrm{mJy})$.

The predicted free-free emission from the thick inner ring is brighter, but still insufficient to account for the ISOCAM detections. To estimate its contribution to the 


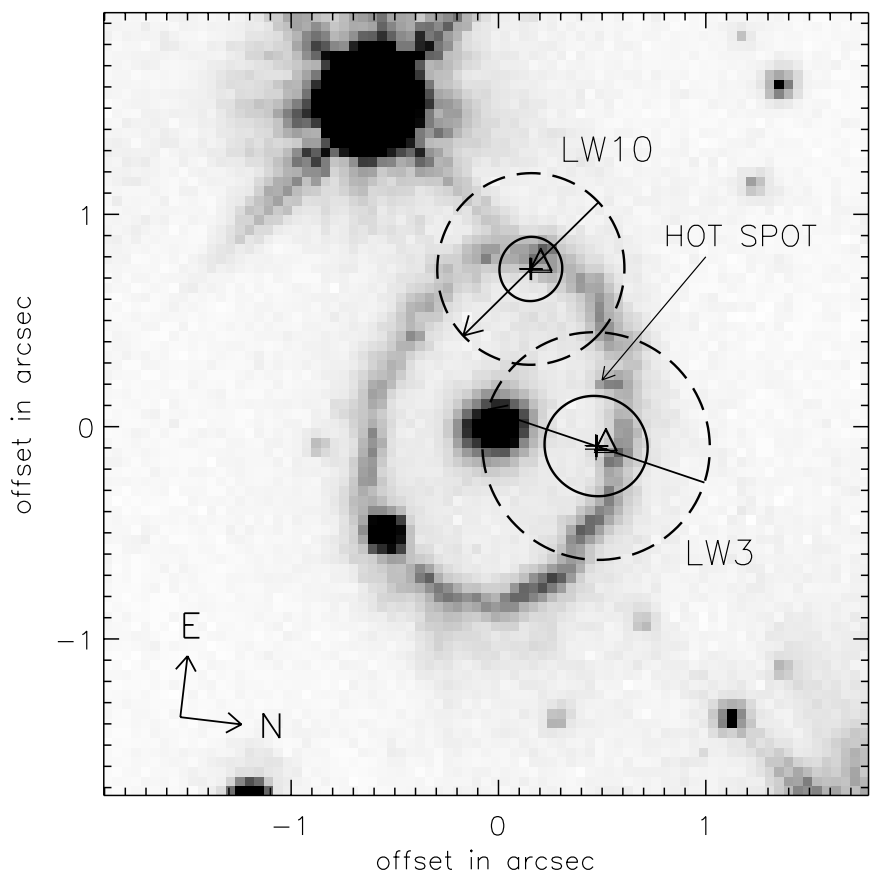

(a) Derived positions of the IR emission

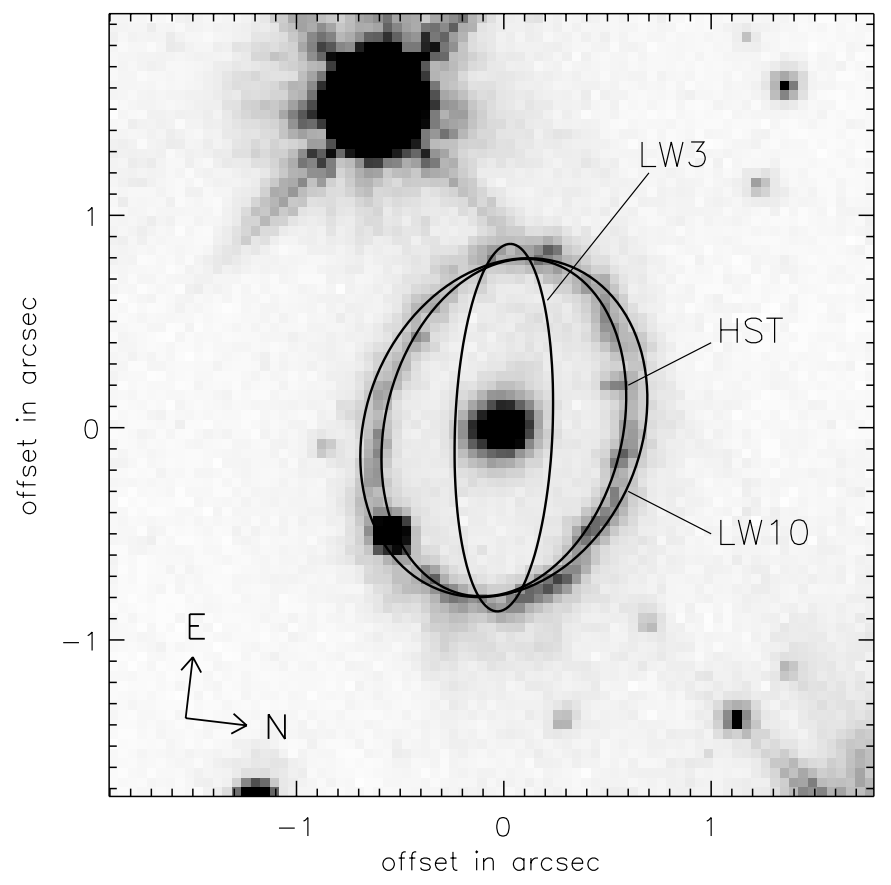

(b) Derived sizes of the IR-emitting region

Fig. 5. Derived absolute coordinates and sizes of the IR-emitting region of the measurements at $12 \mu \mathrm{m}(L W 10)$ and $14.3 \mu \mathrm{m}$ ( $L W 3$ ), overlaid on an HST image of SN 1987A, made in $V$-band with the WFPC2 on 6. February 1996 (showing the thick inner ring and emission from the fast expanding ejecta inside). The point source to the east is an unrelated star (after convention Star 3, e.g. Walborn et al. 1993). The bright spot on the ring to the south-west is a star in the line of sight. a) The coordinates of the infrared emission, derived on the basis of NTT and HST data, are shown as crosses and triangles, respectively. The almost circular ellipses, drawn with a solid and a broken line, respectively, show the statistical errors $\left(\sim 00^{\prime \prime} 15\right.$ and $\sim 00^{\prime \prime} 24$, respectively at 12 and $14.3 \mu \mathrm{m}$ ) and the total inaccuracy of the derived positions of the IR-emission. The systematic error is not well known, because of the edge position of the star, and is simply assumed to be given by 0. .'42, which is the combined uncertainty of the pixel coordinates in the inner ISOCAM field after correcting for distortion (Aussel 1998). The arrows indicate the orientations of the $Z$-axis of the ISO-satellite. Also shown is the position of the so-called Hot Spot, where at least since 1995 the emission in optical wavelengths at the inner boundary of the inner ring started to brighten (Lawrence et al. 2000). b) The derived sizes of the emitting regions for the two IR measurements with the LW10 and LW3-filter are drawn as ellipses centred on the position of SN 1987A. Each of the two axes of the ellipses correspond to the $F W H M$ of the two dimensional Gaussian, determined for the IR-source. The orientation and size of the derived size of the IR-emission at $12 \mu \mathrm{m}$ agrees very well with the projected ellipse of the inner ring in the HST-picture, which is outlined, using the orientation and sizes given by Burrows et al. (1995).

IR the temperature of the whole inner ring was taken to be $10^{4} \mathrm{~K}$ as was derived from analysis of emission spectra, made with the HST at different epochs from a bright part of the inner ring (Lundqvist \& Sonneborn 1997). We used the flux of the inner ring in $R$-band, measured with the WFPC2 of the HST 3268 days after outburst (Soderberg et al. 1999). Further, we assumed that $19 \%$ of the flux is due to the $\mathrm{H}_{\alpha}$-line and the rest to the neighbouring NII lines at 6548 and $6583 \AA$, which is roughly consistent with the line ratios in the line spectrum of a luminous part of the ring presented by Panagia et al. (1996). For the flux density of the free-free emission at $10 \mu \mathrm{m}$ from the inner thick ring we derived an upper limit of $\sim 0.053 \mathrm{mJy}$.

The contribution of the synchrotron radiation, the free-free emission of the ring and the debris to the IR is shown in Fig. 6, which summarizes the spectral energy distribution of the SN 1987A at late times.
No sensitive observations of the MIR cooling lines at late epochs are available. However, according to the theoretical predictions for the evolution of the emission from various fine structure lines of the supernova debris (Kozma \& Fransson 1998a) their contribution should be negligible. We conclude that the measured IR emission is most probably thermal emission from dust. This emission could either be due to grains in the shocked gas in the circumstellar environment or originate in condensates heated mainly by the energy of the radioactive decay of the nucleosynthesis products.

To derive dust masses knowledge of the optical properties of the grains is needed. The grain emissivity can depend quite strongly on grain composition and in particular in the ISOCAM range on wavelength (see e.g. Draine \& Lee 1984). Nevertheless, for a first interpretation the dust spectrum may be approximated by a modified blackbody function of the form already used for the colour correction 


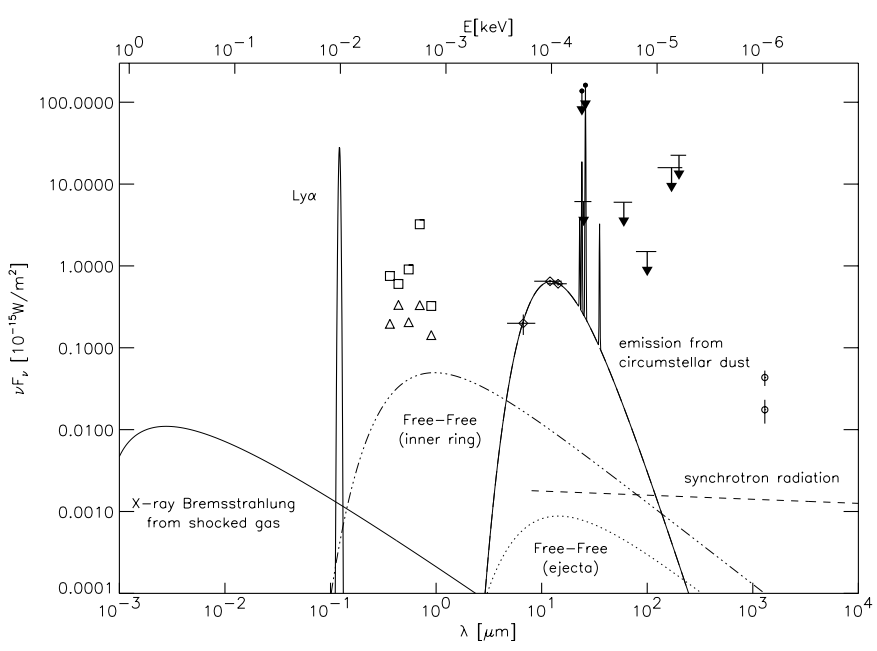

Fig. 6. Spectral energy distribution of the SN 1987A at late times. The measured ISOCAM fluxes are shown as open diamonds. They are colour-corrected according to the modified black body spectrum $F_{\lambda} \propto B_{\lambda}(T) \lambda^{-1}$ with a temperature of $236.1 \mathrm{~K}$ taken as approximation for the circumstellar dust emission (solid line). Upper limits in broad band at 25, 60, 100,170 and $200 \mu \mathrm{m}$ (short horizontal lines) are measurements with ISOPHOT made at the same epoch as the ISOCAM observations (Fischera 2000). The open circles show measured fluxes at $1.3 \mathrm{~mm} 1289$ and 1645 days after outburst (Biermann et al. 1992). The $\mathrm{L} \alpha$ fluxes 3715 and 3734 days after outburst are taken from Sonneborn et al. (1998). Upper limits, derived from measurements with the SWS-spectrometer aboard ISO and shown as filled circles, are taken from Lundqvist et al. (1999). Optical fluxes from the thick inner ring (triangles) and from the debris (squares), derived from observations with the WFPC2 of the HST, are taken from Soderberg et al. (1999). The synchrotron spectrum is an extrapolation from radio measurements, published by Gaensler et al. (1997). For the Bremsstrahlung in X-rays (solid line) we assumed a temperature of $2 \times 10^{7} \mathrm{~K}$ and scaled the emission measure $\sim 2500$ days after outburst $\left(E M=(1.4 \pm 0.4) \times 10^{57} \mathrm{~cm}^{-3}\right.$, Hasinger et al. 1996) with $E M \propto t^{2.06}$ to the time of the ISOCAM observations. The free-free emission of the thick inner ring and the debris are derived from line fluxes, given by Chugai et al. (1997), and the flux in $R$-band, given by Soderberg et al. (1999). The lines in IR are FeII cooling lines from the ejecta at late times derived from theoretical studies for $10^{-4} M_{\odot}$ of ${ }^{44} \mathrm{Ti}$ (taken from Lundqvist et al. 1999). In particular the line at $26 \mu \mathrm{m}$, which is thought to be the strongest cooling line at late epochs, is of basic interest as a tracer of the mass of ${ }^{44} \mathrm{Ti}$. Using this line the $3 \sigma$ upper limit of $51 \mathrm{mJy}$ of the ISOPHOT broad band measurement at $25 \mu \mathrm{m}$ corresponds to a $3 \sigma$ upper limit of $\sim 1.5 \times 10^{-4} M_{\odot}$ for the mass of ${ }^{44} \mathrm{Ti}$ (Fischera 2000). The emission from condensates is expected to be mainly in the FIR (see text).

in Sect. 3.1. The flux density of $N(a)$ dust grains with radius $a$ at a distance $D$ radiating at a certain temperature $T$ is then given by

$F_{\lambda}=N(a) \frac{a^{2}}{D^{2}} \pi B_{\lambda}(T) Q_{\lambda}(a)$

where the emission coefficient is assumed to be $Q_{\lambda}(a)=$ $a \xi \lambda^{-\beta}$ with constant factors $\xi$ and $\beta$. The total luminosity of such a spectrum is given by:

$$
\begin{aligned}
L & =N(a) 4 \pi a^{2} \sigma T^{4}\langle Q(a, T)\rangle, \\
& =N(a) 4 \pi a^{2} \sigma T^{4} \frac{\Gamma(4+\beta) \zeta(4+\beta)}{\Gamma(4) \zeta(4)}\left(\frac{k T}{h c}\right)^{\beta} a \xi,
\end{aligned}
$$

where $k, h, c$ and $\sigma$ are the Boltzmann constant, the Planck constant, the velocity of light and the StefanBoltzmann constant. $\langle Q(T, a)\rangle$ is the Planck-averaged emission coefficient and $\Gamma(x)$ and $\zeta(x)$ are the gammaand the zeta-functions. Whereas the emission behaviour of spherical small grains at long wavelengths can be described with $\beta=2$ at shorter wavelengths a better approximation is given by $\beta=1$. The fit of a spectrum with $\beta=1$ including a colour correction to the flux densities measured with ISOCAM is shown in Fig. 6. The corresponding temperature is $236.0 \pm 11.5 \mathrm{~K}$ and the luminosity of the best fit is $L=2.47 \times 10^{28} \mathrm{~W}$. As seen in the figure the approximation gives a good explanation of the observed colours $\left(\chi_{\min }^{2}=0.14\right)$.

The luminosity can be compared with the rate of deposition of energy from the decay of radioactive decay products. Assuming the ejecta after 11 years to be optically thin to gamma rays and taking an initial mass of $10^{-4} M_{\odot}$ of ${ }^{44} \mathrm{Ti}$ which is mainly responsible for the heating at late epochs then the luminosity emitted by dust grains in the MIR is at least $\sim 20 \%$ of the deposited energy rate $L \approx 1.23 \times 10^{29} \mathrm{~W}$ (Fischera 2000 ).

The dust mass was estimated from the equation

$M_{\mathrm{dust}}=\frac{L \rho}{3 \sigma T^{4}(\langle Q(T, a)\rangle / a)}$,

where $\rho$ is the grain density. We used Planck-averaged emission coefficients at $236 \mathrm{~K}$ of silicate and graphite grains with a radius $a=0.01 \mu \mathrm{m}$ adopted from published values from Laor \& Draine (1993) which are $\left\langle Q_{\mathrm{Si}}\right\rangle / a[\mu \mathrm{m}] \approx 0.42$ and $\left\langle Q_{\mathrm{Gra}}\right\rangle / a[\mu \mathrm{m}] \approx 0.080$. The grain densities are taken to be $2.3 \mathrm{~g} / \mathrm{cm}^{3}$ for graphite and $3.2 \mathrm{~g} / \mathrm{cm}^{3}$ for silicate grains. The masses of silicate and graphite grains are found to be $\sim 1.7 \times 10^{-7} M_{\odot}$ and $\sim 6.8 \times 10^{-7} M_{\odot}$ independent of grain size.

These values indicate a dust mass which is much lower than the minimum dust mass of $\sim 10^{-4} M_{\odot}$ derived for the newly formed dust in the metal rich part of supernova ejecta (Wooden 1997). It is probable that the bulk of the condensates are currently emitting in the FIR/submm and were too cold to be visible to ISOCAM. A temperature of at least $\sim 200 \mathrm{~K}$ is needed to explain the colours of the ISOCAM measurement. By contrast, even as soon as $\sim 1300$ days after outburst, the inferred temperature of condensates was only $\sim 140 \mathrm{~K}$ (Bouchet et al. 1991; Biermann et al. 1992). The condensate temperature should, due to the expansion of the ejecta and weaker heating, have been even lower during the ISOCAM observation $\sim 4000$ days after outburst. The bulk of the condensates should therefore mainly emit in the FIR. Energetically, it is feasible that the observed MIR emission could nevertheless be attributed to a small fraction of condensates 
residing in clumpy regions rich in radioactive nucleosynthesis products where the local heating would be stronger. However, detailed modeling of this case shows that the MIR spectral shape would only be reproduced for a small range of filling factors, composition and sizes (Fischera 2000).

The fact that the MIR-emission was resolved by ISOCAM strongly suggests that the bulk of the detected emission does not arise from condensates. The condensates are thought to be restricted to the central metal rich core of the ejecta (see e.g. Wooden 1997), that is expanding with a velocity of $2000 \mathrm{~km} \mathrm{~s}^{-1}$ (Kozma \& Fransson 1998b). At the time of the ISOCAM observations $\sim 4000$ days after outburst the condensates did not cover a region larger than $\sim 0$.' 18 .

The derived size of $1.50_{-0.24}^{+0.21}$ arcsec and the orientation of the emitting region, with an extension consistent with the elliptical projection of the thick inner ring (Sect. 3.2), both point to a predominantly circumstellar origin for the dust emission. The MIR extent is furthermore consistent with the extensions of the radio (Gaensler et al. 1997) and X-ray (Burrows et al. 2000) emitting regions. The presence of dust in the circumstellar environment has also been concluded from observations of scattered light correlated with the thick inner ring and the associated nebula (Wampler et al. 1990; Crotts et al. 1995).

Due to the fact that both the radio and the X-ray emission are thought to be associated with the blast wave as it propagated into an HII-region of moderate density interior of the thick inner ring (Chevalier \& Dwarkadas 1995), it is likely that the emission in the MIR arises from circumstellar dust heated by shocked gas. The observed dust temperature of $\sim 200 \mathrm{~K}$ is consistent with the predictions for collisionally heated grains in equilibrium with a surrounding hot gas with a density of several $100 \mathrm{~cm}^{-3}$ (Fig. 7 in Dwek 1987). Gas density of this order is indeed inferred from the observed X-ray emission in the shocked circumstellar medium of SN 1987A (Borkowski et al. 1997). It is therefore to be expected that the MIR emission should have a detailed morphology similar to the ring-like structure as seen in X-rays (Burrows et al. 2000) with the centre close to the position of the supernova. The derived luminosity in the MIR is higher than in X-rays (Hasinger et al. 1996) by a factor of $\sim 10$ as seen also in Fig. 6 . This is in agreement with values typically observed in galactic SNRs (Fig. 6 in Dwek 1988).

The presence of dust in the circumstellar environment is consistent with a red supergiant phase in the time evolution of the supernova progenitor (Woosley 1988). In particular, our interpretation of the IR emission is qualitatively in agreement with the hypothesis that the HIIregion is wind material from this red supergiant phase as suggested by Chevalier \& Dwarkadas (1995).

\section{Summary}

We have presented measurements made with ISOCAM at $6.75,12$ and at $14.3 \mu \mathrm{m} \sim 11$ years after outburst of
SN 1987A. To characterize the IR source we derived the absolute coordinates and analysed the extension of the emitting region.

1. The flux densities of the MIR source associated with the supernova at $6.75,12$ and at $14.3 \mu \mathrm{m}$ are $0.496 \pm$ $25.1 \% \mathrm{mJy}, 2.41 \pm 4.3 \% \mathrm{mJy}$ and $2.82 \pm 5.5 \% \mathrm{mJy}$ (respectively 3855.2, 3998.7 and 3937.7 days after outburst) (Sect. 3).

2. The offsets of the IR emission of the remnant of $\mathrm{SN} 1987 \mathrm{~A}$ at $12(L W 10)$ and at $14.3 \mu \mathrm{m}(L W 3)$ from the position of the supernova $\left(\alpha=5^{\mathrm{h}} 35^{\mathrm{m}} 27.968^{\mathrm{s}}\right.$, $\delta=-69^{\circ} 16^{\prime} 11^{\prime \prime}$.09 (J2000), Reynolds et al. 1995), derived relative to an IR-emitting star in the ISOCAM fields (see Sect. 3.3) are found to be:

$$
\begin{aligned}
& \Delta \alpha=-0^{\prime \prime} \cdot 04 \pm 0^{\prime \prime} \cdot 24_{\text {stat. }} \pm 0^{\prime \prime} \cdot 42_{\text {sys. }} \\
& \Delta \delta=+0^{\prime \prime} 48 \pm 0^{\prime \prime} \cdot 24_{\text {stat. }} \pm 0 . \prime 42_{\text {sys. }}
\end{aligned}
$$

and

$$
\begin{aligned}
& \Delta \alpha=+0 . .77 \pm 0.15_{\text {stat. }} \pm 0 . \prime 42_{\text {sys. }} \text {, } \\
& \Delta \delta=+0 . .09 \pm 0 . \text { ' } 15_{\text {stat. }} \pm 0 . ! 42_{\text {sys. }} \text {. }
\end{aligned}
$$

Due to the edge position of the star the uncertainty of the measurement at $12 \mu \mathrm{m}$ may be larger. On the basis of the absolute coordinates taken in isolation the MIR emission could be associated either with the supernova or the circumstellar interaction region seen at radio and X-ray wavelengths.

3. The absolute coordinates of the IR-emitting star derived using observations of the SN 1987A made with HST and NTT are (with a $1 \sigma$ uncertainty of $\sim 00^{\prime \prime} 09$ ): $\alpha=5^{\mathrm{h}} 35^{\mathrm{m}} 18.418^{\mathrm{s}}, \delta=-69^{\circ} 16^{\prime} 30^{\prime \prime} 65$.

4. From the observation at $12 \mu \mathrm{m}$ the size of the IRemitting region, assuming a spherical symmetric Gauss function for the source, is found to be $1.50_{-0.24}^{+0.21}$ arcsec $(F W H M)$, consistent with the diameter of $1.66 \pm$ 0.03 arcsec found by Panagia et al. (1991) for the thick inner ring. Further analysis of these IR data with an elliptical Gauss function also shows a remarkably good agreement of the IR-emitting region with the orientation and the extension of the projected ring even though the result is not very significant. The measured extension is a clear indication of a predominantly circumstellar origin for the IR emission. In particular the emission from condensates is not prominent, because the condensates would be restricted after 4000 days to a region with a diameter of $\sim 0$ ". 18 .

Acknowledgements. The work was supported by Deutsches Zentrum für Luft- und Raumfahrt e.V. (DLR) through the projects "50 OR 9702" and "50 OR 99140". The ISOCAM data presented in this paper were analysed using "CIA", a joint development by the ESA Astrophysics Division and the ISOCAM Consortium. The ISOCAM Consortium is led by the ISOCAM PI, C. Cesarsky. The paper is based on observations made with ESO Telescopes at the La Silla or Paranal Observatories under program ID 000.0-0000 (observation day: 10/01/95, observer: $\mathrm{S}$. Benetti). It is further based on observations made with the 
NASA/ESA Hubble Space Telescope, obtained from the data archive at the Space Telescope Institute. STScI is operated by the association of Universities for Research in Astronomy, Inc. under the NASA contract NAS 5-26555. We thank René Gastaud, Stephan Ott, Leo Metcalf and Ralf Siebenmorgen for discussions and information relevant to the analysis of the ISOCAM data. We thank the referees for their helpful suggestions.

\section{Appendix A: Theoretical resolution function}

To estimate the flux, size and coordinates of the IR-source we made use of a theoretical point spread function ( $p s f$ ) for the ISO-satellite kindly made available to us by Dr. Ralph Siebenmorgen. Images of point sources observed in narrow band $L W$-filters of ISOCAM have been shown to be (except for $L W 1$ ) in good agreement with this function, which we will refer to as the monochromatic psf (Okumura 1998). For the broad-band filters the $p s f$ depends however on the source spectrum. Therefore to achieve a high accuracy in the determination of the source extension we used a theoretical broad-band $p s f$, given by:

$J_{\mathrm{F}}(\boldsymbol{x})=\frac{\int \mathrm{d} \lambda \lambda R(\lambda) J(\boldsymbol{x}, \lambda)}{\int \mathrm{d} \lambda \lambda R(\lambda) F_{\lambda}}$.

Here $R(\lambda)$ is the spectral transmission of an ISOCAM filter, taken from the CIA program and $F_{\lambda}$ the flux density (assumed to have a power law $\left.F_{\lambda} \propto \lambda^{-k}\right) . J(\boldsymbol{x}, \lambda)$ is given by

$J(\boldsymbol{x}, \lambda)=\frac{A F_{\lambda}}{\lambda^{2}} \operatorname{psf}(\boldsymbol{x}, \lambda)$,

where $A$ is the effective area of the telescope and $p s f$ the monochromatic point spread function scaled to unity at $\boldsymbol{x}=0 . J(\boldsymbol{x}, \lambda)$ was derived on a grid of $2048 \times 2048$ grid points sampled at $1 / 3^{\prime \prime} \times 1 / 3^{\prime \prime}$ using a FFT technique. We used values of $60 \mathrm{~cm}, 17.4 \mathrm{~cm}$ and $2 \mathrm{~cm}$ for the diameter of the primary mirror, the borehole and the thickness of the braces of the tripod, respectively.

Finally, we convolved the obtained function $J_{\mathrm{F}}(\boldsymbol{x})$ with the used pixel size and the probability distribution of the actual pointing position of the satellite during the observation (jitter).

To measure source extensions smaller than the resolution of the instrument it is important to know how accurately the theoretical psf represents the actual psf, particularly in the kernel. To this end we compared the $F W H M$ of the theoretical ps $f$ of the $L W 10$ and the $L W 3$ filters (for spectral index $k=4$ ) with the actual $F W H M$ derived from observations of point sources. The $F W H M$ of cuts in $Y$ and $Z$ (in satellite coordinates) through the peak of the observed brightness distribution was found to be in agreement to within $1 \%$ with the $F W H M$ of the theoretical psf. A corresponding comparison between Gaussian fits to the observed point source and the theoretical psf also agrees within 1\% (Table A.1). The asymmetry of the true $p s f$ in the first ring visible in the images of point-like sources (Okumura 1998) should have only a very minor effect.
Table A.1. FWHM of the measured and the theoretical psf.

\begin{tabular}{l||c|c}
\hline \hline Filter & $L W 10$ & $L W 3$ \\
\hline \hline \multicolumn{3}{c||}{$F W H M$ of cuts through the maximum } \\
\hline$\left.F W H M_{\text {meas. }} .^{\prime \prime}\right]^{a}$ & $3.981 \pm 0.084^{b}$ & $4.980 \pm 0.012$ \\
$F W H M_{\text {meas. }}\left[^{\prime \prime}\right]^{c}$ & $3.951 \pm 0.252$ & $4.980 \pm 0.063$ \\
$F W H M_{\text {theor. }}\left[^{\prime \prime}\right]$ & $3.945 \pm 0.026$ & $4.956 \pm 0.058$ \\
\hline relation meas./theor. ${ }^{d}$ & $1.009 \pm 0.022$ & $1.005 \pm 0.005$ \\
\hline \multicolumn{3}{c}{$F W H M$ of a Gaussian approximation } \\
\hline$\left.F W H M_{\text {meas. }} .^{[\prime}\right]^{c}$ & 4.071 & 4.896 \\
$F W H M_{\text {theor. }}\left[{ }^{\prime \prime}\right]$ & 4.035 & 4.866 \\
\hline relation meas. $/$ theor. & 1.009 & 1.006 \\
\hline
\end{tabular}

a Values taken from Okumura (1998).

$b$ The given uncertainties correspond to the difference in the two values derived in $Y$ and $Z$ direction.

${ }^{c}$ Derived from observations of HIC 78527 ( $\left.L W 10\right)$ and HIC 80331 ( LW3).

${ }^{d}$ Due to values from Okumura.

An uncertainty of less than $1 \%$ is smaller than the effect of varying the spectral shape in the calculation in the psf. For Gaussian approximations to the theoretical psf we found that the dependence of the $F W H M$ on the spectral index for the $L W 3$ and $L W 10$ filters is close to linear. In a broad range from $k \approx-4$ to $k \approx 4$ the $F W H M$ is approximately given by (Fischera 2000):

$$
\begin{array}{ll}
F W H M(k)=4^{\prime \prime} .349-0^{\prime \prime} 081 k & (L W 10), \\
F W H M(k)=5^{\prime \prime} 078-0^{\prime \prime} 048 k & (L W 3) .
\end{array}
$$

If the theoretical ps $f$ of the calibration star $(k=4)$ would also be taken for the IR emission from the supernova remnant (which in reality has $k \approx 1$ ) the $F W H M$ of the $p s f$ would be underestimated by $\sim 6 \%$ and $\sim 3 \%$ for the $L W 10$ and $L W 3$ filters, respectively. This can cause substantial systematic uncertainties in the derived source sizes (Fig. 3; Sect. B.2).

\section{Appendix B: Method for determination of the probability distribution in source size}

To derive the probability distributions shown in Fig. 3 we measured the variation of $\chi^{2}\left(\sigma_{\mathrm{Q}}\right)$ as function of the variance $\sigma_{\mathrm{Q}}$ of the symmetrical Gauss function assumed for the source. We also left flux density $\left(F_{\mathrm{Q}}\right)$ and coordinates $\left(\boldsymbol{x}_{0}\right)$ as free variables. Because the source is smaller than the resolution of the telescope, we had to convolve the source function with the theoretical psf described in the previous section. To transform the variation of $\chi^{2}$ into a probability distribution we approximated the $p s f$ itself through a two dimensional symmetrical Gaussian function with variance $\sigma_{\mathrm{J}}$. The value of $\sigma_{\mathrm{J}}$ was found through a simple $\chi^{2}$-fit between the $p s f$ and the Gaussian. Measuring the most probable size $\sigma_{\mathrm{Q}_{0}}$ of the source is then equivalent to measuring the size of the image with the variance 
$s_{0}=\sqrt{\sigma_{\mathrm{Q}_{0}}^{2}+\sigma_{\mathrm{J}}^{2}}$. In the case of normal distributed errors the probability distribution of $s$ with

$s=\sqrt{\sigma_{\mathrm{Q}}^{2}+\sigma_{\mathrm{J}}^{2}}$

is a Gaussian distribution with the variance $\sigma_{\mathrm{s}}$ :

$p(s)=\frac{1}{\sqrt{2 \pi} \sigma_{\mathrm{s}}} \exp \left(-\frac{1}{2}\left(\frac{s-s_{0}}{\sigma_{\mathrm{s}}}\right)^{2}\right)$

so that the variation of $\Delta \chi^{2}(s)$ is a simple quadratic function:

$\Delta \chi^{2}(s)=\chi^{2}(s)-\chi^{2}\left(s_{0}\right)=\frac{1}{\sigma_{\mathrm{s}}^{2}}\left(s-s_{0}\right)^{2}$,

where $\chi^{2}\left(s_{0}\right)=\chi_{\text {min }}^{2}(s)$. The measured variation of $\chi^{2}\left(\sigma_{\mathrm{Q}}\right)$ can therefore be approximated by:

$\chi^{2}\left(\sigma_{\mathrm{Q}}\right)=\frac{1}{\sigma_{\mathrm{s}}^{2}}\left(\sqrt{\sigma_{\mathrm{Q}}^{2}+\sigma_{\mathrm{J}}^{2}}-s_{0}\right)^{2}+\chi^{2}\left(s_{0}\right)$.

The most probable value $s_{0}$, its uncertainty $\sigma_{\mathrm{s}}$ and the corresponding value of $\chi^{2}\left(s_{0}\right)$ we derived again through a $\chi^{2}$-fit. Due to uncertainties in the model and the data the value $s_{0}$ can also be smaller than $\sigma_{\mathrm{J}}$.

To get the probability distribution over the physical sizes of the source we normalized the distribution with:

$N=\int_{\sigma_{\mathrm{J}}}^{\infty} \mathrm{d} s p(s)=\frac{1}{2}\left(1-\operatorname{erf}\left(\frac{\sigma_{\mathrm{J}}-s_{0}}{\sqrt{2} \sigma_{\mathrm{s}}}\right)\right)$

using the error function:

$\operatorname{erf}(y)=\frac{2}{\sqrt{\pi}} \int_{0}^{y} \mathrm{~d} t \mathrm{e}^{-t^{2}}$

The confidence levels of the maximum sizes of the source are simply given by:

$C L\left(\sigma_{\mathrm{Q}}\right)=\frac{1}{2 N}\left(\operatorname{erf}\left(\frac{s-s_{0}}{\sqrt{2} \sigma_{\mathrm{s}}}\right)-\operatorname{erf}\left(\frac{\sigma_{\mathrm{J}}-s_{0}}{\sqrt{2} \sigma_{\mathrm{s}}}\right)\right)$

where $s$ is given by Eq. (B.1.)

\section{B.1. Accuracy of the method}

The accuracy of the used method to derive the extension of the emitting region can be estimated by assuming that IR source and $p s f$ are given by Gauss functions with variances $\sigma_{\mathrm{Q}}$ and $\sigma_{\mathrm{J}}$. An uncertainty $\Delta \sigma_{\mathrm{J}}$ in the variance of the $p s f$ limits a measurement of the extension, neglecting uncertainties in the data, to sources that are larger than $\sigma_{\mathrm{Q}} \approx \sqrt{2 \Delta \sigma_{\mathrm{J}} \sigma_{\mathrm{J}}}$. The uncertainties in the $F W H M$ of the theoretical psf as stated in Table A.1 gives a resolution limit of $\sim 0$.' $6(F W H M)$ for both filters as will be verified in the next section.
Table B.1. Observation log of the calibration stars.

\begin{tabular}{l||c|c}
\hline \hline Filter & $L W 10$ & $L W 3$ \\
\hline target & HIC 78527 & HIC 80331 \\
TDT & 189007 & 119036 \\
observer & CAM CAL & CAM CAL \\
observation date & 15.3 .1996 & 24.5 .1996 \\
raster & $6 \times 6$ & $6 \times 6$ \\
pixel size & $3^{\prime \prime}$ & $3^{\prime \prime}$ \\
step size & $2^{\prime \prime}$ & $2^{\prime \prime}$ \\
read out interval & $0.28 \mathrm{~s}$ & $0.28 \mathrm{~s}$ \\
time between pointings & $60 \mathrm{~s}$ & $60 \mathrm{~s}$ \\
\hline
\end{tabular}

\section{B.2. Verification of the method using calibration stars}

To verify the method for measuring the angular size of the MIR emission associated with SN 1987A we did two independent tests. Firstly, we made a control experiment by applying the same method to observations of pointlike source calibration stars (Sect. B.2.1). Secondly, we repeated the procedure on the supernova data, but using the image of a calibration star as the psf in place of the theoretical psf (see Sect. B.2.2). The results both show that the model of the theoretical psf allows a measurement of the size of a Gaussian source with a precision which corresponds to an inaccuracy of less than $\sim 1 \%$ in the $F W H M$. For the two filters we derived a resolution limit of about $0^{\prime \prime} 6$ at $95 \%$ confidence level.

As measurements of point-like sources we have chosen for LW3 and LW10 filters observations of HIC 78527 and of HIC 80331, respectively. As in the case of SN 1987A the observation provides a sampling of the source with an angular resolution of $1^{\prime \prime}$ with a pixel size of $3^{\prime \prime}$ (Table. B.1). The data were calibrated in an almost identical way as the observation of SN 1987A with the LW2 filter. The only difference was that the deglitching for the calibration star data could be done fully automatically using standard procedures due to the benign radiation environment encountered for these observations. From the data we first derived four images with a sampling of $1^{\prime \prime}$ and calculated the final one as a weighted average.

\section{B.2.1. Extension measurements for the calibration stars}

The probability distributions in source size of the calibration stars were derived from background-subtracted images using theoretical $p s f$ s for a spectral index of $k=4$ appropriate for the Rayleigh-Jeans part of the spectrum. For the calculation we used a subimage of $11 \times 11$ pixels centred on the source.

The result is shown in Fig. B.1. As for the supernova data we have taken the uncertainties of the pixel values to be equal and assigned a value such that the minimum $\chi^{2}$ corresponds to a confidence level of $90 \%$. The most probable sizes are found to be much smaller than the $F W H M$ of the $p s$. For both filters we found that the source is smaller 

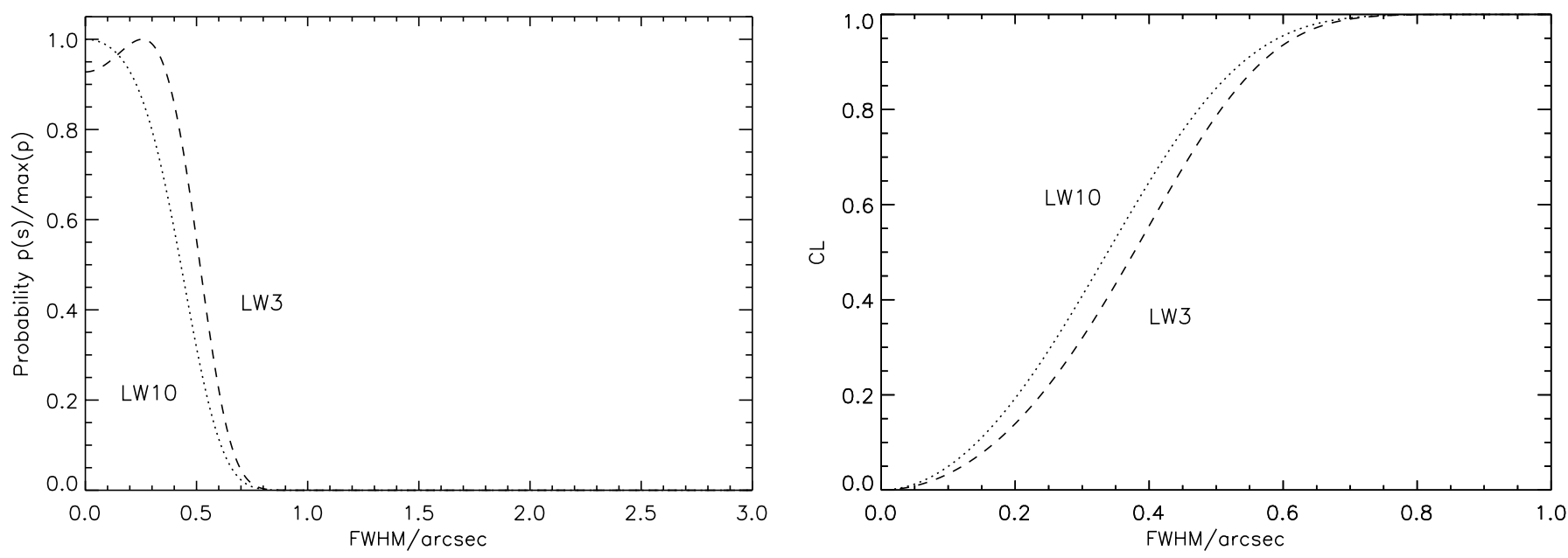

Fig. B.1. Result of the size measurement for the point-like sources HIC 80331 ( $L W 3$-filter) and HIC 78527 ( $L W 10$-filter). The derived probability distributions of the size $s$ (see Eq. (B.1)) of the theoretical image $B$ (Eq. (2)) as a function of the $F W H M$ of the Gaussian source is shown in the left panel. The right panel gives the corresponding confidence levels for upper limits on source size derived using Eq. (B.7).

than $\sim 0^{\prime \prime} 6$ with a confidence level of $95 \%$. This is consistent with the rough estimate of $0{ }^{\prime \prime} 6$ given in Sect. B.1.

\section{B.2.2. Comparison of the size measurements of the MIR emission using observed and theoretical psfs}

No point-like calibration source with the same spectrum of the source associated with SN 1987A was observed with ISOCAM. However, the effect of interchanging the theoretical $p s f$ with the observed $p s f$ could still be evaluated. This was done by comparing calculations for the size probability distributions at $12 \mu \mathrm{m}$, respectively using as psf the observed image of a star and a theoretical ps $f$ for a spectral index $k=4$. To achieve for the image of the calibration star the same sampling of $1 / 3^{\prime \prime}$ as for the theoretical psf we used a cubic interpolation applied to the logarithm of the pixel values. It can be seen from Fig. 3 that a most probable size of 1 ". 9 is derived both for the measured and the theoretical psf. The larger size derived by using the measured image of a calibration star instead of the theoretical psf appropriate for the supernova is caused by the steeper spectrum.

\section{Appendix C: Position of the reference star}

To determine the position of the IR-emitting star we used archival observations with the WFPC2 of the HST, made in $V$-band in February 1996 and three observations (two in $R$ one in $V$-band) made in 1995 of the supernova with the SUSI-instrument (SUperb-Seeing Imager) of the NTT (New Technology Telescope). The $V$-band filter was also used to determine the correction of the distortion on the WFPC2-fields (Holtzman et al. 1995). The measurement with the NTT was necessary due to the fact that the supernova and the IR-emitting star were on different CCDs of the observation with the WFPC2, which can give an additional error in the astrometry. The astrometry on one single CCD of the WFPC2 is accurate to $0 \prime 1$. The only object, whose coordinates are determined with high accuracy is the SN 1987A itself. To remove the offset in the astrometry of the WFPC2 data, we have taken the position $\alpha=5^{\mathrm{h}} 35^{\mathrm{m}} 27.968^{\mathrm{s}}, \delta=-69^{\circ} 16^{\prime} 11^{\prime \prime}$. 09 (J2000) from Reynolds et al. (1995), which is accurate to $\sim 70 \times 10^{-3}$ arcsec. We removed the distortion on the WFPC2-field using the polynomial correction given by Holtzman et al. (1995).

To derive the astrometry of the observations made with the SUSI instrument, we projected all possible star positions, seen on a single CCD of the WFPC2, with a tangential projection on the SUSI field and compared these coordinates $\left(x_{i}, y_{i}\right)$ with the star positions $\left(\tilde{x}_{i}, \tilde{y}_{i}\right)$ on the SUSI field with a non linear $\chi^{2}$-fit:

$$
\chi^{2}=\sum_{i} \frac{\left(\left(x_{i}-\tilde{x}_{i}\right)^{2}+\left(y_{i}-\tilde{y}_{i}\right)^{2}\right)^{2}}{\sigma^{2}}
$$

The positions of the individual stars we derived fitting a two dimensional symmetrical Gauss function to the single stars in the fields.

We found no indication of a distortion on the SUSI field. The final derivations of the star positions in the observations with the SUSI instrument and the WFPC2 were accurate by $\sigma=0^{\prime \prime} .025$ in the case of the PC-field of the WFPC2 and by $\sigma=00^{\prime \prime} 049$ in the case of the CCD with the star (WF3). This is consistent with the accuracy given for the single CCDs of the WFPC2 after correcting for distortion $\left(\sigma \approx 0^{\prime \prime} 04\right.$, Holtzman et al. 1995). The single CCDs indeed show a small offset of up to $\sim 0^{\prime \prime} 13$. On the other hand we found no measurable offset for the two CCDs with the supernova and the single IR-emitting star. The absolute coordinates of the single IR-emitting star in the SUSI field are found to be

$\alpha=5^{\mathrm{h}} 35^{\mathrm{m}} 18.42^{\mathrm{s}}, \quad \delta=-69^{\circ} 16^{\prime} 30^{\prime \prime} 65$. 
The coordinates directly derived from the WFPC2 observation only differed by $\sim 0$.'04 in right ascension and by $\sim 0$.'05 in declination. The position of the star relative to the SN 1987A should be accurate to $\sim 00^{\prime \prime} 05$. The uncertainty of the absolute coordinates should be $\sim 0$. 09 .

\section{References}

Arnett, W. D., Bahcall, J. N., Kirshner, R. P., \& Woosley, S. E. 1989, ARA\&A, 27, 629

Aussel, H. 1998, ISOCAM LW Channel Field of View Distortion, Version 0.9, Uncomplete Draft

Biermann, P. L., Chini, R., Haslam, C. G. T., et al. 1992, A\&A, $255, \mathrm{~L} 5$

Blommaert, J. 1998, ISOCAM PHOTOMETRY REPORT

Blommaert, J., \& Cesarsky, D. 1998, ISOCAM CALIBRATION ACCURACIES DOCUMENT

Blommaert, J., Siebenmorgen, R., Coulais, J., et al. 1999, ISO Handbook Volume III (CAM)

Borkowski, K. J., Blondin, J. M., \& McCray, R. 1997, ApJ, 476, L31

Bouchet, P., Danziger, I. J., \& Lucy, L. B. 1991, AJ, 102, 1135

Burrows, C. J., Krist, J., Hester, J. J., et al. 1995, ApJ, 452, 680

Burrows, D. N., Michael, E., Hwang, U., et al. 2000, ApJ, 543, L149

Cesarsky, C. J., et al. 1996, A\&A, 315, L32

Chalabaev, A. A., Perrier, C., \& Mariotti, J.-M. 1989, A\&A, 210, L1

Chevalier, R. A., \& Dwarkadas, V. V. 1995, ApJ, 452, L45

Chugai, N. N., Chevalier, R. A., Kirshner, R. P., \& Challis, P. M. 1997, ApJ, 483, 925

Crotts, A.P.S., Kunkel, W. E., \& Heathcote, S. R. 1995, ApJ, 438,724

Draine, B. T., \& Lee, H. M. 1984, ApJ, 285, 89

Draine, B. T., \& Salpeter, E. E. 1979, ApJ, 231, 438

Dwek, E., \& Scalo, J. M. 1980, ApJ, 239, 193

Dwek, E. 1987, ApJ, 322, 812

Dwek, E. 1988, Infrared Analysis of Supernova Remnants in Supernovae Remnants and the Interstellar Medium, IAU Coll. 101, ed. R. S. Roger, \& T. L. Landecker

Fischera, Jg. 2000, Infrarotemission der SN 1987A nach 11 Jahren, Thesis, Heidelberg

Gaensler, B. M., Manchester, R. N., Staveley-Smith, L., et al. 1997, ApJ, 479, 845

Gould, A. 1995, ApJ, 452, 189

Gould, A., \& Uza, O. 1998, ApJ, 494, 118

Hasinger, G., Aschenbach, B., \& Trümper, J. 1996, A\&A, 312, L9

Holtzman, J. A., Hester, J. J., Casertano, S., et al. 1995, PASP, 107,156

Hoyle, F., \& Wickramasinghe, N. C. 1970, Nature, 226, 62
Jones, A. P., Tielens, A. G. G. M., Hollenbach, D.J., \& McKee, C. F. 1994, ApJ, 433, 797

Kessler, M. F. et al. 1996, A\&A, 315, L27

Kozma, C., \& Fransson, C. 1998a, ApJ, 497, 431

Kozma, C., \& Fransson, C. 1998b, ApJ, 496, 946

Laor, A., \& Draine, B. T. 1993, ApJ, 402, 441

Lawrence, S. S., Sugerman, B. E., Bouchet, P., et al. 2000, ApJ, 537, L123

Lundqvist, P., \& Sonneborn, G. 1997, in The Structure of the Circumstellar Gas of SN 1987A in SN 1987A, Ten Years After, proceedings, ed. M. Phillips, \& N. Suntzeff

Lundqvist, P., Sollerman, J., Kozma, C., et al. 1999, A\&A, 347, 500

Okumura, K. 1998, ISOCAM PSF Report

Martin, P. G. 1988, ApJS, 66, 125

Ott, S., et al. 1998, Astronomical Data Analysis Software and Systems VII, ASP Conf. Ser. 145, ed. R. Albrecht, R. N. Hook, \& H. A. Bushouse, 275

Panagia, N., Gilmozzi, R., Macchetto, F., et al. 1991, ApJ, 380, L23

Panagia, N., Scuderi, S., Gilmozzi, R., et al. 1996, ApJ, 459, L17

Press, W. H., Teukolsky, S. A., Vetterling, W. T., \& Flannery, B. P. 1992, in Numerical Recipes in C

Rank, D. M., Bregman, J., Witteborn, F. C., et al. 1988, ApJ, 325, L1

Reynolds, J. E., Jauncey, D. L., \& Staveley-Smith, L. 1995, A\&A, 304, 116

Roche, P. F., Aitken, D. K., \& Smith, C. H. 1993, MNRAS, 261,522

Schubert, J. 1995, technical report, Max-Planck-Institut für Astronomie

Shull, J. M. 1977, ApJ, 215, 805

Soderberg, A. M., Challis, P., \& Suntzeff, N. B. 1999, Amer. Astron. Soc. Meet., 194, \#86.12

Sonneborn, G., Fransson, C., Lundqvist, P., et al. 1997, ApJ, 477,848

Sonneborn, G., Pun, C. S. J., Kimble, R. A., et al. 1998, ApJ, 492, L139

Tielens, A. G. G. M., McKee, C. F., Seab, C. G, \& Hollenbach, D. J. 1994, ApJ, 431, 321

Tuffs, R. J., Fischera, Jg., Drury, L.O'C, et al. 1998, The Universe as seen by ISO, Paris, France, 20.-23. Oct. 1998

Walborn, N. R., Phillips, M. M., Walker, A. R., \& Elias, J. H. 1993, PASP, 105, 1240

Wampler, E. J., Wang, L., Baade, D., et al. 1990, ApJ, 362, L13

Wooden, D. H. 1997, Observational Evidence for Mixing and Dust Condensation in Core-Collapse Supernovae, in Astrophysical Implications of the Laboratory Study of Presolar Materials, AIP Conf. Proc. 402, ed. T. J. Bernatowicz \& E. Zinner

Woosley, S. E. 1988, ApJ, 330, 218 